\title{
Colloid (mucinous non-cystic) carcinoma of the pancreas: A case report
}

\author{
YANG GAO, YA-YUN ZHU and ZHOU YUAN \\ Department of General Surgery, Shanghai Jiao Tong University Affiliated to Sixth People's Hospital, \\ Shanghai 200233, P.R. China
}

Received January 6, 2015; Accepted September 4, 2015

DOI: 10.3892/ol.2015.3733

\begin{abstract}
Colloid carcinoma (CC) of the pancreas, also known as mucinous non-cystic carcinoma, is a rare histological variant of pancreatic cancer. The present study reports the case of a 65 -year-old male with a pancreatic head tumor $4.6 \times 3.1 \mathrm{~cm}$ in size. The tumor had not invaded the superior mesenteric or celiac arteries. Laboratory data showed elevated alkaline phosphatase, $\gamma$-gluytamyl transpeptidase and cancer antigen 19-9 (CA19-9) levels. The patient underwent a pancreaticoduodenectomy (PD), and the pathology revealed $\mathrm{CC}$ extending to the submucosa of the duodenum, with mild atypical hyperplasia of the pancreatic duct. The patient was negative for circulating tumor cells, indicating a good prognosis. The CA19-9 concentration decreased to within the normal range following surgery. The present study reports a rare case of CC of the pancreas presenting with obstructive jaundice, in which a PD procedure was performed. Primary surgical treatment with curative intent is the optimal management and this subtype of pancreatic cancer is associated with a better prognosis compared with pancreatic ductal adenocarcinoma.
\end{abstract}

\section{Introduction}

Colloid carcinoma (CC) of the pancreas, also known as mucinous non-cystic carcinoma, is a rare subtype of pancreatic cancer that only accounts for only $1-3 \%$ of the malignant neoplasms of the exocrine pancreas. The incidence of $\mathrm{CC}$ is suspected to be only a few cases per 1 million individuals per year $(1,2)$. Although CC and pancreatic ductal adenocarcinoma (PDAC) share similar clinical manifestations, $\mathrm{CC}$ represents a disparate clinical entity.

$\mathrm{CC}$ is a histological variant of ductal adenocarcinoma with unique pathological and clinical characteristics that differ

Correspondence to: Professor Zhou Yuan, Department of General Surgery, Shanghai Jiao Tong University Affiliated to Sixth People's Hospital, 600 Yishan Road, Shanghai 200233, P.R. China

E-mail: zhouyuan851@163.com

Key words: colloid carcinoma, circulating tumor cell, diagnose, prognose, pancreaticoduodenectomy from normal ductal adenocarcinoma. Pathologically, CC is described as well-circumscribed pools of abundant stromal mucin with floating elements, including malignant cells that account for $50 \%$ of the tumor volume (3). However, the mucin produced in the pools prevents the spread of the malignant cells, acting as a physical barrier and protracting the disease course of CC (4). Open biopsy may lead to the dissemination of the tumor due to the adherent nature of the mucin; thus, the advantages and disadvantages of open biopsy should be carefully considered during fine needle aspiration biopsy (5). Clinically, computed tomography (CT) and magnetic resonance imaging (MRI) findings may be useful for determining the correct differential diagnosis, allowing optimal treatment planning and prognosis to be achieved $(6,7)$. The available treatment regimens are similar to those of normal ductal adenocarcinoma. For example, surgical resection is recommended in symptomatic patients with low surgical risk (1). Notably, these CCs almost always arise from intraductal papillary mucinous neoplasms (IPMN), and CC has a significantly better prognosis than that of pancreatic ductal adenocarcinoma, according to the literature $(1,8)$.

The current study presents a case of CC in a 65-year-old male with an $\sim 3$-week history of malaise and decreased appetite. Written informed consent was obtained from the patient.

\section{Case report}

In September 2014, a 65-year-old male with an 3-week history of weakness, malaise and decreased appetite was transferred to the Department of General Surgery, Shanghai Jiao Tong University Affiliated to Sixth People's Hospital (Shanghai, China), and the patient's weight decreased by $10 \mathrm{~kg}$ within 1 month. The patient was first referred to the Department of Infectious Disease (Shanghai Jiao Tong University Affiliated to Sixth People's Hospital) in August 2014 due to a history of chronic hepatitis B infection for 30 years and a history of treatment with entecavir for 5 years. The patient exhibited no abdominal pain, nausea or vomiting. On physical examination, palmar erythema was present, but abdominal examination revealed no tenderness or rebound tenderness. The patient had no family history of malignancy. Liver function tests suggested obstructive jaundice [total bilirubin, $16.1 \mu \mathrm{mol} / 1$ (normal range, $0.0-18.0 \mu \mathrm{mol} / \mathrm{l}$ ); conjugated bilirubin, $9.7 \mu \mathrm{mol} / 1$ (normal range, 0.0-6.0 $\mu \mathrm{mol} / 1$ ); alkaline phosphatase, $308 \mathrm{U} / 1$ (normal range, 15-112 U/l); and 

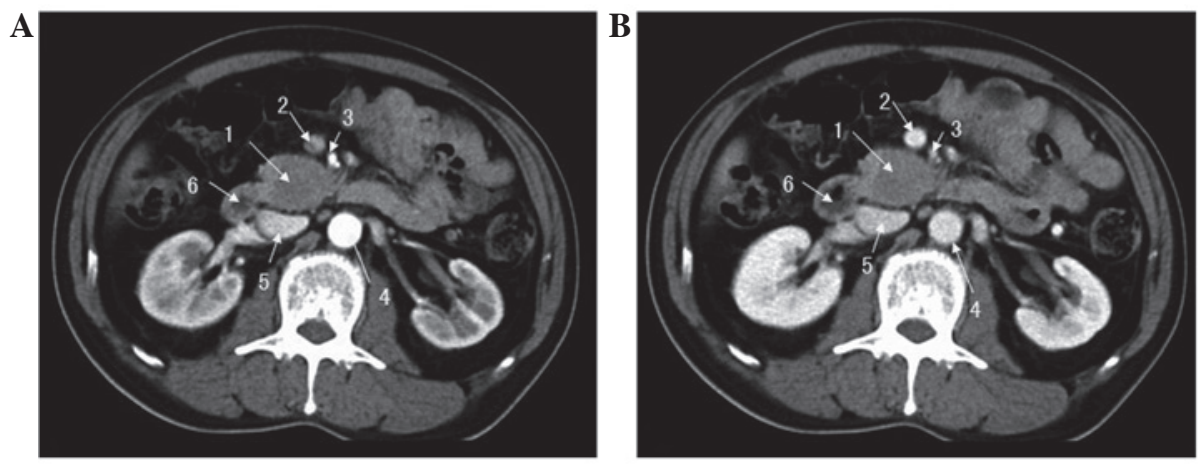

Figure 1. Abdominal computed tomography with contrast at (A) artery and (B) venous phases, revealing a mass located in the uncinate process of the pancreas. 1, Pancreatic mass; 2 , superior mesenteric vein; 3 , superior mesenteric artery; 4 , abdominal aorta; 5 , inferior veno cava; 6 , duodenum.
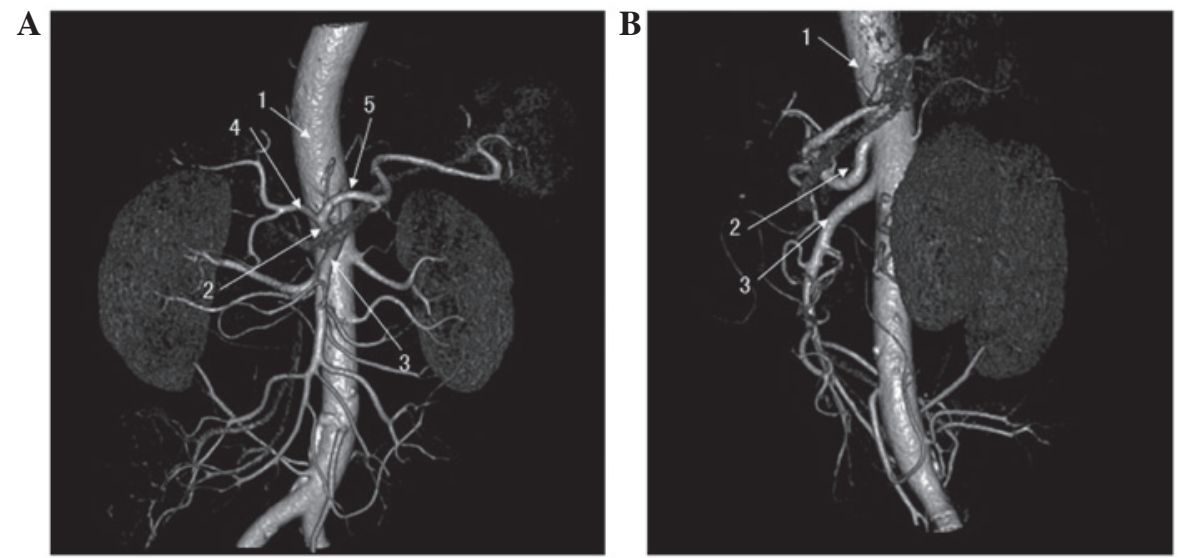

Figure 2. Abdominal artery computed tomography in the (A) anterior and (B) lateral views, showing that the mass had not invaded the superior mesenteric artery and celiac artery. 1, Abdominal aorta; 2 , celiac artery; 3 , superior mesenteric artery; 4 , common hepatic artery; 5 , splenic artery.

A

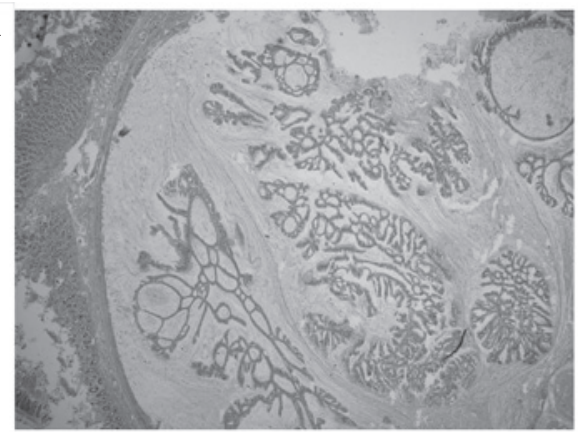

B

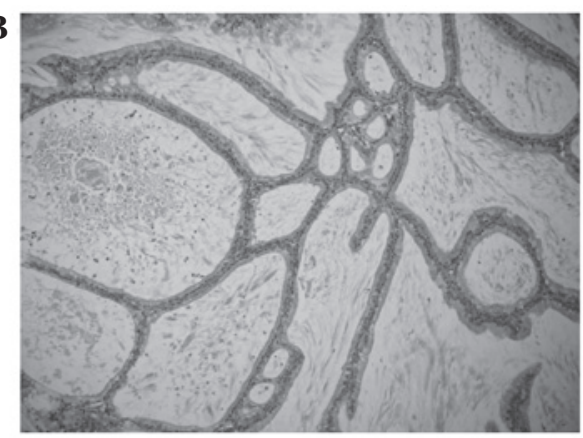

Figure 3. Pathological examination. (A) Examination of the specimen showing invasion of colloid carcinoma into the duodenal wall (hematoxylin and eosin staining; magnification, x100). (B) Mucin pools with floating clumps and strands of malignant tumor cells were present. The mucin pools were lined by cuboidal, well-differentiated epithelium (hematoxylin and eosin staining; magnification, x200).

$\gamma$-gluytamyl transpeptidase $887 \mathrm{U} / 1$ (normal range, 0-50 U/1)] An abnormal tumor marker level of cancer antigen 19-9 (CA19-9; $57.65 \mathrm{U} / \mathrm{ml}$; normal range, 0.00-27.00 U/ml) was detected, while the level of carcinoembryonic antigen (CEA) was normal (7.67 ng/ml; normal range, 0.00-10.00 ng/ml). An enhanced CT scan of the upper abdomen revealed a hypovascular mass measuring $4.6 \times 3.1 \mathrm{~cm}$ within the uncinate process of the pancreas, but the boundary between the tumor and the horizontal region of the underlying duodenum was obscure. The result of the abdominal artery CT was consistent with the enhanced CT, and it also indicated that the mass had not invaded the adjacent arteries, such as the superior mesenteric artery (SMA) and celiac artery. Therefore, pancreatic head cancer was suspected pre-operatively and a pancreaticoduodenectomy (PD) was decided upon for this patient. Prior to surgery, $7.5 \mathrm{ml}$ of peripheral blood was drawn to detect the circulating tumor cells (CTCs) to assess the tumor metastasis and prognosis.

During the surgery, a solid $4 \times 3-\mathrm{cm}$ tumor was noted, without significant invasion to the adjacent tissues. The mass was well-circumscribed, with no invasion of the SMA, celiac artery or inferior vena cava, and regional enlarged lymph nodes were not noted. The tumor was located in the pancreatic head. 
Therefore, the PD procedure was performed and a digestive tract reconstruction was performed via an end-to-end pancreaticojejunostomy, an end-to-side hepaticojeunostomy and an end-to-side gastrojejunostomy. The post-operative course was uneventful and the level of CA19-9 decreased to within the normal range $(19.75 \mathrm{U} / \mathrm{ml})$ at day 7 post-surgery. The patient was negative for circulating tumor cells. The patient was discharged from hospital within 10 days of surgery.

Finally, the histopathological analysis of the resected specimen revealed the mucinous non-cystic carcinoma (CC) of the pancreas, measuring $2.5 \times 2 \mathrm{~cm}$, extending to the submucosa of the duodenum, with mild atypical hyperplasia of the pancreatic duct. According to the American Joint Committee on Cancer tumor-node-metastasis staging of pancreatic cancer (2010), the tumor was T3N0M0 (stage IIA) (9). Immunohistochemical analysis showed positivity for cytokeratin 7, Ki-67 (15\%), human epidermal growth factor receptor-2, p53 and CA19-9, but negative results for CEA and CA-125.

\section{Discussion}

$\mathrm{CC}$ of the pancreas is a histopathological variant of pancreatic cancer in which mucin accounts for at least $50 \%$ of the tumor, according to the World Health Organization (10). Large mucin pools with floating mucin-producing tumors, including signet-ring cells, are present $(1,8)$. CC and IPMNs are usually abundant in mucin 2, oligomeric mucus/gel-forming (MUC2) glycoprotein, but no mucin 1, cell surface-associated (MUC1) glycoprotein, while PDAC is characterized by strong expression of MUC1, but lacks MUC2 (11-13). Additionally, MUC2 exhibits tumor suppressor activity, therefore playing a vital role in the progression of IPMN and CC for relatively low invasive potential. The pancreatic carcinomas with mucinous phenotype (MUC2 ${ }^{+} / \mathrm{MUC1}^{-}$) usually do not exhibit mutations in the mismatch repair genes and are microsatellite stable. This indicates why the malignancy of CC and IPMN is weaker than that of PDAC $(1,14)$.

The clinical manifestations caused by $\mathrm{CC}$ of the pancreas are similar to those of PDAC; the symptoms are indolent and usually present as abdominal pain, jaundice, weight loss and an abdominal mass (2). The incidence of CC appears to exhibit no gender predominance and the median age at first presentation has been reported as 59-69 years old (1). Tumor biomarkers, including CEA and CA19-9, are also elevated. The patient was negative for CTCs in the present case, as detected by the subtraction enrichment technology and immuno-fluorescence in situ hybridization (iFISH). By contrast, patients are usually positive for CTCs in confirmed PDAC cases in the Department of General Surgery of Shanghai Jiao Tong University Affiliated to Sixth People's Hospital. The negative results indicated a good prognosis for the present case, while positive results would have indicated metastasis and the possibility of recurrence in advance of imaging examination (15).

$\mathrm{CT}$ and MRI are useful approaches in the pre-operative diagnosis of patients with CC. CC on CT appears as masses with round or lobular margins, and the tumors usually have clear boundaries. The exception to this is tumors with ill-defined boundaries with the duodenum, which indicates invasion to the duodenum (7). On MRI, CC presents as a mass with lobulating contours and indiscrete margins. The tumors exhibit a hyperintense salt-and-pepper-like appearance on T2-weighted images, and the distinctive MRI features on these images are useful for forming the correct pre-operative diagnosis (6). Furthermore, fine-needle aspiration also plays a pivotal role in the evaluation of $\mathrm{CC}$, and large amounts of mucin and benign-appearing glandular epithelium or single cells can be observed. However, the differential diagnosis of a mucinous tumor is hard to complete by FNA, and the procedure may promote the spread of the primary tumor (16).

$\mathrm{CC}$ of the pancreas is located predominantly in the head of the pancreas, and usually originates from intestinal-type IPMN. Certain CCs involve the tail of the pancreas, and may originate from mucinous cystic neoplasms (1). The diameter of CC ranges between 1.2 and $16.0 \mathrm{~cm}$, which is greater than that of tubular ductal adenocarcinoma at presentation $(1,8)$. Microscopic features of $\mathrm{CC}$ reveal that the tumors consist of separating pools containing mucin and floating clumps or strands of malignant cells; the mucin pools are lined in part by the cuboidal or well-differentiated epithelium (10). Pathological examination of the tumor should enhance our understanding of the inherent characteristics of CC.

No specific guidelines exist for the treatment of $\mathrm{CC}$ at present. The mainstay treatment should be surgery if there is no distant metastasis, surrounding organ invasion or vessel encasemen. Surgeries, such as the Whipple procedure, a distal pancreatectomy, a pylorus-preserving PD and a subtotal pancreatectomy, can be performed on patients with CC (1). The type of the surgery is determined by the location and size of the tumor. Furthermore, chemotherapy and/or the radiation therapy do not significantly improve post-operative survival $(2,17)$. The prognosis of CC of the pancreas has been shown to be better than that of PDAC, with a 5-year survival rate of $>55 \%$ versus $\sim 10 \%$, respectively, and certain patients with lymph node metastasis who remain alive and free of disease after 10 years $(5,13)$. This improved prognosis is suspected to result from the fact that the mucin originating from the drafting neoplasm cells in the pool and the epithelial cells with the secretary properties limit the tumor cell spread (4).

In summary, $\mathrm{CC}$ of the pancreas is a rare subtype of pancreatic cancer. The clinical manifestations of $\mathrm{CC}$ are similar to those of PDAC, however, the histopathology shows quite unique features, including a mucinous pool lined with cuboidal, well-differentiated epithelium cells and containing drafting clumps or strands of neoplastic cells. Notably, the patient was negative for CTCs in the present case, indicating a good prognosis. Curative resection is the optimal treatment and could lead to full remission.

\section{Acknowledgements}

The authors would like to thank Dr Juan Tang for her expertise in immunohistochemistry and photography.

\section{References}

1. Liszka L, Zielinska-Pajak E, Pajak J and Gołka D: Colloid carcinoma of the pancreas: Review of selected pathological and clinical aspects. Pathology 40: 655-663, 2008.

2. Whang EE, Danial T, Dunn JC, Ashley SW, Reber HA, Lewin TJ and Tompkins RK: The spectrum of mucin-producing adenocarcinoma of the pancreas. Pancreas 21: 147-151, 2000. 
3. Baker ML, Seeley ES, Pai R Suriawinata AA, Mino-Kenudson M, Zamboni G, Klöppel G and Longnecker DS: Invasive mucinous cystic neoplasms of the pancreas. Exp Mol Pathol 93: 345-349, 2012.

4. Plerhoples TA, Ahdoot M, DiMaio MA, Pai RK, Park WG and Poultsides GA: Colloid carcinoma of the pancreas. Dig Dis Sci 56: 1295-1298, 2011.

5. Reid MD, Bagci P and Adsay NV: Histopathologic assessment of pancreatic cancer: Does one size fit all? J Surg Oncol 107: 67-77, 2013.

6. Yoon MA, Lee JM, Kim SH, Lee JY, Han JK, Choi BI, Choi JY, Park SH and Lee MW: MRI features of pancreatic colloid carcinoma. AJR Am J Roentgenol 193: W308-W313, 2009.

7. Ren FY, Shao CW, Zuo CJ and Lu JP: CT features of colloid carcinomas of the pancreas. Chin Med J (Engl) 123: 1329-1332, 2010.

8. Adsay NV, Pierson C, Sarkar F, Abrams J, Weaver D, Conlon KC Brennan MF and Klimstra DS: Colloid (mucinous noncystic) carcinoma of the pancreas. Am J Surg Pathol 25: 26-42, 2001.

9. Edge SB and Compton CC: The American Joint Committee on Cancer: The 7th Edition of the AJCC cancer staging manual and the future of TNM. Ann Surg Oncol 17: 1471-1474, 2010.

10. Kloppel G, Hruban RH, Longnecker DS, Adler G, Kern SE and Partanen TJ: Ductal adenocarcinoma of the pancreas. In: World Health Organization Classification of Tumors. Pathology and Genetics of Tumors of the Digestive System. Hamilton SR and Aaltonen LA (eds). IARC Press, France, pp221-230, 2000.

11. Luttges $\mathbf{J}$ and Kloppel G: Update on the pathology and genetics of exocrine pancreatic tumors with ductal phenotype: Precursor lesions and new tumor entities. Dig Dis 19: 15-23, 2001
12. Lüttges J, Zamboni G, Longnecker D and Klöppel G: The immunohistochemical mucin expression pattern distinguishes different types of intraductal papillary mucinous neoplasms of the pancreas and determines their relationship to mucinous noncystic carcinoma and ductal adenocarcinoma. Am J Surg Pathol 25: 942-948, 2001.

13. Adsay NV, Merati K, Nassar H, Shia J, Sarkar F, Pierson CR, Cheng JD, Visscher DW, Hruban RH and Klimstra DS: Pathogenesis of colloid (pure mucinous) carcinoma of exocrine organs: Coupling of gel-forming mucin (MUC2) production with altered cell polarity and abnormal cell-stroma interaction may be the key factor in the morphogenesis and indolent behavior of colloid carcinoma in the breast and pancreas. Am J Surg Pathol 27: 571-578, 2003.

14. Lüttges J, Beyser K, Pust S, Paulus A, Rüschoff J and Klöppel G Pancreatic mucinous noncystic (colloid) carcinomas and intraductal papillary mucinous carcinomas are usually microsatellite stable. Mod Pathol 16: 537-542, 2003.

15. Zhang Y, Wang F, Ning N, Chen Q, Yang Z, Guo Y, Xu D, Zhang D, Zhan T and Cui W: Patterns of circulating tumor cells identified by CEP8, CK and CD45 in pancreatic cancer. Int J Cancer 136: 1228-1233, 2015

16. Parwani AV and Ali SZ: Pathologic quiz case: A 52-year-old woman with jaundice and history of necrotizing pancreatitis. Primary colloid carcinoma of the pancreas. Arch Pathol Lab Med 129: 255-256, 2005.

17. Rubio-Perez I, Martin-Perez E, Sanchez-Urdazpal L, Corbaton P and Larrañaga E: Colloid carcinoma of the pancreas: A distinct pancreatic neoplasm with good prognosis. Report of a case. JOP 13: 219-221, 2012. 